\title{
Serum MRGPRX2 as a long-time available biomarker for drug-induced anaphylaxis
}

\author{
Jin $\mathrm{An}^{1}$ and Tae-Bum Kim ${ }^{2}$ \\ ${ }^{1}$ Kyung Hee University Hospital at Gangdong \\ ${ }^{2}$ Asan Medical Center
}

November 18, 2020

\section{Serum MRGPRX2 as a long-time available biomarker for drug-induced anaphylaxis}

To the Editor,

The diagnosis of anaphylaxis is based on clinical symptoms after exposure to a trigger factor ${ }^{1}$. Serum tryptase is useful for an accurate diagnosis of anaphylaxis. However, one of its limitations is short half-life, hence the need for blood samples to be obtained at the onset of anaphylaxis ${ }^{2,3}$. Mas-related G proteincoupled receptor-X2 (MRGPRX2), expressed in mast cells, is related to degranulation of mast cells in the IgE-independent pathway for anaphylaxis ${ }^{4-6}$. We aimed to evaluate serum MRGPRX2 levels in patients with drug-induced anaphylaxis (DIA) and to determine usefulness as a potential marker of anaphylaxis by maintaining its concentrations longer.

We included 68 patients who consulted at allergy clinic for a history of anaphylaxis after causal drug administration between January 2010 and December 2017. Diagnosis of DIA and causal drugs were determined by an allergist. Patients were excluded if 1) causal drugs were ambiguous, or 2) other relevant causes were combined. Each patient underwent serum MRGPRX2 level measurement randomly at least once from the onset of anaphylaxis. MRGPRX2 levels were determined using an ELISA kit from MyBioSource. We also measured MRGPRX2 levels of 74 controls.

We classified DIA patients according to causal drugs to investigate the difference in MRGPRX2 levels between drugs. In addition, we analyzed the MRGPRX2 levels according to the time interval from the anaphylactic event to verify maintenance of MRGPRX2 levels over a longer period of time. The time interval for each patient was defined as the gap between the measurement time of MRGPRX2 levels and the anaphylactic event. This study was approved by the institutional review board of Asan Medical Center (2018-1252), and subjects provided written informed consent.

This study included 74 controls and 68 patients with DIA. The mean MRGPRX2 level in the DIA group was significantly higher than that of the control group (57.4 \pm 42.8 vs. $17.5 \pm 12.7, p<.001)$ (Figure $1 \mathrm{~A})$. Patients were classified into 5 subgroups according to their causal drugs, including antibiotics, non-steroidal antiinflammatory drugs (NSAIDs), histamine-2 receptor blockers (H2- blockers), radiocontrast media (RCM), and others. The others included clopidogrel, proton pump inhibitors, diltiazem, carboplatin, rifampicin, and ethambutol. The most common causal drug was antibiotics (49\%), followed by NSAIDs (19\%), and H2 blockers (10\%). The mean MRGPRX2 levels in patients with DIA caused by antibiotics were higher than in those with other subgroups, but there was no significant difference between the 5 causal drugs $(p=0.964)$ (Table 1).

MRGPRX2 levels of patients with $4-5$ week time interval were higher $(97.4 \pm 39.7)$ than those with $0-1$ week (51.9 \pm 45.2$), 1-2$ weeks $(54.0 \pm 42.1), 2-3$ weeks $(66.9 \pm 39.7)$, and $3-4$ weeks $(47.2 \pm 27.6)$ but with no significant 
difference $(p=0.099)$ (Table 1). Overall, mean MRGPRX2 levels in each time interval were higher than those in the controls (Figure 1B). There was no significant difference among the frequencies of the causal drugs in each time interval (Table S1).

This study demonstrated that serum MRGPRX2 was a potential long-time biomarker for DIA, maintaining high concentration for more than a month from the onset of anaphylaxis. Patients with DIA had higher mean MRGPRX2 levels than controls in all time intervals from the anaphylaxis onset. Thus, MRGPRX2 may be a promising biomarker for supporting a diagnosis of DIA over a longer period than tryptase.

This study has limitations in that there was no data of serial changes in MRGPRX2 concentration according to the time interval in the same patient and no direct comparison between MRGPRX2 and tryptase levels. However, this is a pilot study. The performance of MRGPRX2 as a useful biomarker of DIA needs to be further studied using a larger population and in a longer time.

\section{References}

1. Simons FE. Anaphylaxis pathogenesis and treatment. Allergy.2011;66 Suppl 95:31-34.

2. Lee AYS. Elevated Serum Tryptase in Non-Anaphylaxis Cases: A Concise Review. Int Arch Allergy Immunol. 2020;181:357-364.

3. Platzgummer S, Bizzaro N, Bilo MB, et al. Recommendations for the use of tryptase in the diagnosis of anaphylaxis and clonal mastcell disorders. European Annals of Allergy and Clinical Immunology.2020;52:5161.

4. McNeil BD, Pundir P, Meeker S, et al. Identification of a mast-cell-specific receptor crucial for pseudoallergic drug reactions. Nature. 2015;519:237-241.

5. Ali H. Emerging Roles for MAS-Related G Protein-Coupled Receptor-X2 in Host Defense Peptide, Opioid, and NeuropeptideMediated Inflammatory Reactions. G Protein-Coupled Receptors in Immune Response and Regulation. 2017;136:123-162.

6. Subramanian H, Gupta K, Ali H. Roles of Mas-related G protein-coupled receptor X2 on mast cellmediated host defense, pseudoallergic drug reactions, and chronic inflammatory diseases. Journal of Allergy and Clinical Immunology. 2016;138:700-710.

\section{Conflict of interest}

The authors declare no conflicts of interest.

\section{Acknowledgments}

This research was supported by the Bio \& Medical Technology Development Program of the National Research Foundation (NRF) funded by the Korean government (MSIT) (2019M3E5D3073365).

\section{Keywords}

Adverse drug reaction, drug-induced anaphylaxis, tryptase, MRGPRX2, biomarker

Jin $\mathrm{An}, \mathrm{MD}, \mathrm{PhD}{ }^{1,2}$

Tae-Bum Kim MD, $\mathrm{PhD}^{1}$

${ }^{1}$ Department of Allergy and Clinical Immunology, Asan Medical Center, University of Ulsan College of Medicine, Seoul, Republic of Korea

${ }^{2}$ Department of Pulmonary, Allergy and Critical Care Medicine, Kyung Hee University Hospital at Gangdong, College of Medicine, Kyung Hee University, Seoul, Republic of Korea

\section{Correspondence}


Tae-Bum Kim, MD, PhD

Department of Allergy and Clinical Immunology, Asan Medical Center,

University of Ulsan College of Medicine, Seoul, Republic of Korea

Tel: 82-2-3010-3280; Fax: 82-2-3010-6969

E-mail: tbkim@amc.seoul.kr

Table 1. Clinical characteristics and serum MRGPRX2 levels in drug-induced anaphylaxis patients.

\begin{tabular}{lll}
\hline & MRGPRX2 level $(\mathrm{ng} / \mathrm{mL})$ & $P$ value \\
\hline Age & & 0.711 \\
$<40$ years $(\mathrm{n}=22)$ & $54.6 \pm 43.2$ & $? \dot{\imath} ?$ \\
40 years $(\mathrm{n}=46)$ & $58.7 \pm 43.1$ & 0.540 \\
Sex & & \\
Male $(\mathrm{n}=27)$ & $61.3 \pm 49.9$ & \\
Female $(\mathrm{n}=41)$ & $54.8 \pm 37.9$ & 0.918 \\
Causal drugs & & \\
Antibiotics $(\mathrm{n}=33)$ & $61.4 \pm 43.7$ & \\
NSAIDs $(\mathrm{n}=13)$ & $48.3 \pm 38.5$ & \\
H2-blockers $(\mathrm{n}=7)$ & $54.0 \pm 33.8$ & \\
RCM $(\mathrm{n}=5)$ & $54.1 \pm 75.6$ & \\
Others ${ }^{+}(\mathrm{n}=10)$ & $59.9 \pm 37.3$ & \\
Time interval from anaphylaxis event & & \\
$0-1$ week $(\mathrm{n}=31)$ & $51.9 \pm 45.2$ & \\
$1-2$ weeks $(\mathrm{n}=16)$ & $54.0 \pm 42.1$ & \\
$2-3$ weeks $(\mathrm{n}=7)$ & $66.9 \pm 39.7$ & \\
$3-4$ weeks $(\mathrm{n}=8)$ & $47.2 \pm 27.6$ & \\
$4-5$ weeks $(\mathrm{n}=6)$ & $97.4 \pm 39.7$ & \\
\hline
\end{tabular}

NSAIDs, non-steroidal anti-inflammatory drugs; H2-blockers, histamine-2 receptor blockers; RCM, radiocontrast media +Others included clopidogrel, proton pump inhibitors, diltiazem, carboplatin, rifampicin, and ethambutol.

\section{Figure legends}

Figure 1. Differences in MRGPRX2 levels between healthy controls and drug-induced anaphylaxis (DIA) (A). Mean serum MRGPRX2 level of the DIA $(57.4 \pm 42.8)$ was significantly higher than that of healthy controls $(17.5 \pm 12.7)(p<.001)$. Higher MRGPRX2 levels were observed in patients with DIA regardless of time interval from anaphylaxis compared to controls depicted as the dotted line (B). The MRGPRX2 levels of each time interval from anaphylaxis are as follows: $51.9 \pm 45.2$ (0-1 week), $54.0 \pm 42.1$ (1-2 weeks), $66.9 \pm 39.7$ (2-3 weeks), 47.2 \pm 27.6 (3-4 weeks), and $97.4 \pm 39.7$ (4-5 weeks).

\section{Supplement}

\section{Statistical analysis}

Differences in MRGPRX2 levels in healthy controls and patients with DIA were calculated using Student's t tests. Differences in clinical variables between groups were evaluated using chi-square tests. One-way analysis of variance (ANOVA) was applied to analyze the difference in MRGPRX2 levels according to causal drugs and time interval. Data were analyzed using MedCalc (version 15.5) statistical software. Two-sided $p$ value of $<0.05$ was considered significant.

Supplementary Table 1 . Frequency of causal drugs in each time interval from anaphylaxis. 


\begin{tabular}{lllllll}
\hline & Antibiotics $(\mathrm{n}=33)$ & NSAIDs $(\mathrm{n}=13)$ & H2-blockers $(\mathrm{n}=7)$ & $\mathrm{RCM}(\mathrm{n}=5)$ & Others $^{+}(\mathrm{n}=10)$ & $P$ value \\
\hline $0-1$ week (n) & 12 & 5 & 3 & 5 & 6 & 0.084 \\
$1-2$ weeks (n) & 10 & 3 & 1 & 0 & 2 & 0.595 \\
$2-3$ weeks (n) & 5 & 1 & 0 & 0 & 1 & 0.678 \\
$3-4$ weeks (n) & 2 & 3 & 2 & 0 & 1 & 0.264 \\
$4-5$ weeks (n) & 4 & 1 & 1 & 0 & 0 & 0.703 \\
\hline
\end{tabular}

NSAIDs, non-steroidal anti-inflammatory drugs; H2-blockers, histamine-2 receptor blockers; RCM, radiocontrast media +Others included clopidogrel, proton pump inhibitors, diltiazem, carboplatin, rifampicin, and ethambutol.

Figure 1
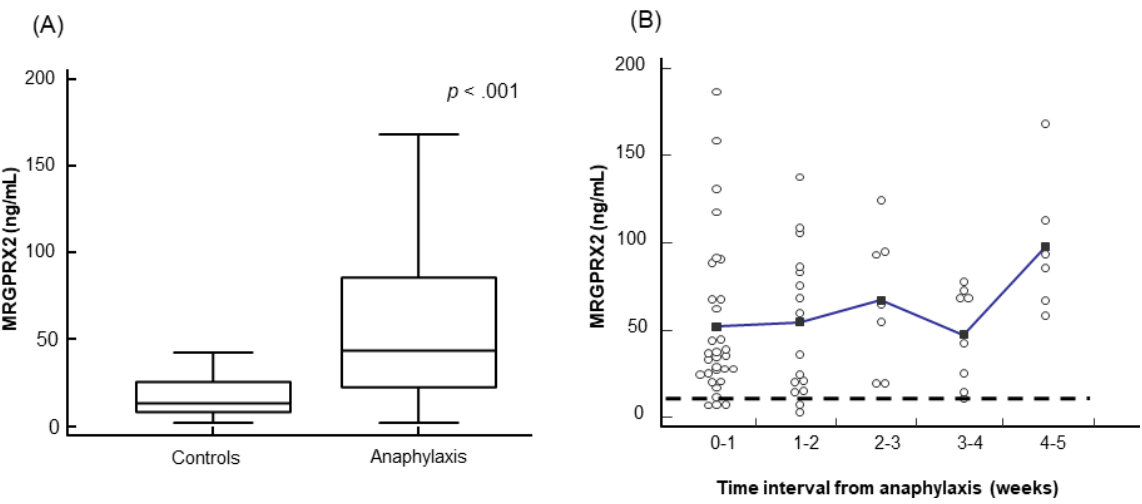